\title{
Animal Husbandry, Dairy and Veterinary Science
}

\section{A novel LAMP field test for the diagnosis of contagious agalactia caused by Mycoplasma agalactiae}

\author{
Loria GR ${ }^{1}$, Puleio R ${ }^{1}$, Nicholas RAJ ${ }^{2 *}$, Manno $\mathrm{C}^{4}$, Arcoleo $\mathrm{G}^{3}$ and Drago $\mathrm{C}^{5}$ \\ ${ }^{1}$ Istituto Zooprofilattico Sperimentale della Sicilia “A. Mirri”-Via G. Marinuzzi, Palermo, Italy \\ ${ }^{2}$ The Oaks, Nutshell Lane, Farnham, Surrey GU9 0HG, UK \\ ${ }^{3}$ Enbiotech, Via Aquileia, Palermo, Italy \\ ${ }^{4}$ Avantech Group, via Masuccio Salernitano, Angri, Italy \\ ${ }^{5}$ Department of Biological, Chemical and Pharmaceutical Sciences and Technologies University of Palermo, Italy
}

\begin{abstract}
The aim of the work was to develop a new, robust, portable diagnostic system based on the latest development of loop-mediated isothermal amplification (LAMP) technology for the detection of the main causative agent of contagious agalactia, an important disease of small ruminants. The integrated diagnostic system consists of a portable instrument and a kit, specific for the pathogen. The kit is ready to use and provides both the reagents for a rapid nucleic acid extraction from milk, and the amplification reagents using LAMP technology, The test showed a high sensitivity identifying only M.agalactiae DNA with a detection limit of less than 100 $\mathrm{CFU} / \mathrm{ml}$ in milk samples, exceeding the traditional laboratory diagnostics for speed and handling The test also proved to be more tolerant to the inhibitory effect of milk components that affect amplification in the PCR. These preliminary results show that the LAMP system can be a practical and effective diagnostic tool for the diagnosis in the field of M.agalactiae pathogen.
\end{abstract}

\section{Introduction}

Contagious agalactia (CA) is caused by four different pathogenic mycoplasmas: Mycoplasma agalactiae, the pathogen with the highest prevalence in Mediterranean areas accounting for over $80 \%$ of diagnoses, followed by M. mycoides sub. capri; M. capricolum subsp. capricolum and $M$. putrefaciens [1]. CA is today one of the top priorities for the sheep and goat livestock sector due to the large losses in milk production in ewes, the production and poor survival of weak lambs, low fertility, vaccine costs and indirect expenses such as treatment, time and labour needed for the management of the disease.

Rapid, low-cost, and user-friendly strategies are urgently needed for early diagnosis of disease and timely treatment, particularly for on-site screening of pathogens of animals often at great distances from diagnostic laboratories. The different diagnostic tests currently used for CA fail to provide sufficiently rapid responses. Serological tests like ELISA are not able to detect specific antibodies until at least 25-30 days by ELISA [2]. The excretion of the antigen itself in infected milk is only detectable by culture from the 9th day of infection onwards to which must be added the additional days, sometimes even weeks, for in vitro isolation [3,4]. More recently, the polymerase chain reaction (PCR) has come into common use due to its ability to increase the speed of response, sensitivity and specificity of the diagnosis [5,6]. However, it remains an established fact that the extraction of the pathogenic DNA from the milk is not always easy due to the natural inhibitors present in the sample that hinders their extraction. In practice, it is often observed that samples of milk with low levels of antigen, as occurs in bulk milk, may be falsely negative when tested. The PCR also has critical factors that exclude its use in the field including the need for a well-equipped laboratory and expensive reagents.
The aim of the work was to develop a new, robust, portable diagnostic system (ICGene ${ }^{\varpi}$ ) based on the latest evolution of loopmediated isothermal amplification (LAMP) technology which reduces all the classical phases of molecular biology, overcoming the use of expensive laboratory equipment needed for traditional or real timePCR. The new method provides results directly in the field in about 1 hour with automatic interpretation of the results.

\section{Material and method}

\section{Sensitivity}

For the sensitivity test, freshly collected sheep milk was used, originating from a flock free of contagious agalactia, and distributed in 8 tubes of $10 \mathrm{ml}$, to the first of which was added $10 \%(1 \mathrm{ml}$ in $9 \mathrm{ml}$ of milk) of culture of Mycoplasma agalactiae (NCTC reference strain code 10123 ) at maximum growth ( 1 X10 $0^{8}$ colony forming units (CFU) / mL) in mycoplasma culture broth (Mycoplasma broth, Hayflick, Oxoid ${ }^{\circ}$ ). Then one $\mathrm{ml}$ of milk was taken from the first test tube and diluted in the subsequent one at a rate of $1 / 10$ until it was estimated that $10 \mathrm{CFU}$ of mycoplasma remained in the last tube. The exact amount of antigen was tested in parallel with viable colony counts on mycoplasma agar [7].

\section{Specificity}

In parallel, four sheep milk tubes each containing other pathogenic mycoplasmas M. putrefaciens, M. m. capri, M. capricolum and the

${ }^{\star}$ Correspondence to: Nicholas RAJ, The Oaks, Nutshell Lane, Farnham, Surrey GU9 0HG, UK, E-mail: robin.nicholas@btinternet.com

Key words: field test, LAMP, contagious agalactia, small ruminants

Received: July 16, 2018; Accepted: July 25, 2018; Published: July 30, 2018 
closely related bovine pathogen $M$. bovis were prepared and tested in order to evaluate the specificity of the method.

The integrated diagnostic system consists of a portable instrument and a kit, specific for the pathogen (Figure 1). The kit is ready to use and provides both the reagents for a rapid nucleic acid extraction starting from $1 \mathrm{ml}$ of milk, and the amplification reagents using LAMP technology.

Six primers were used for the p40 gene (Rekha V. et al., 2015): F3 (external forward primer) GGTTTATTAACTGCGTCATCA; B3 (complementary external primer) CAACAGTTGCATTCGTCTT; FIP (Forward inner primer) CCTTATCACCATTATCTTGTGGATCAGTGCCTTTATTAG; BIP (Backward Inner Primer)ATTAGGTGAAGTTGTCAAAAATATTGAGCTTGCTTCAGGAATTCTGCTA; LF (Forward Loop Primer) GTGAATTTTCGTTCTTATCATCAC; LB (Backward Loop Primer) ACAAATCTAGGTGAAATAGTATTACC.

\section{Result}

The test showed a detection limit of less than $100 \mathrm{CFU} / \mathrm{ml}$ in artificially infected milk samples, comparable with the traditional

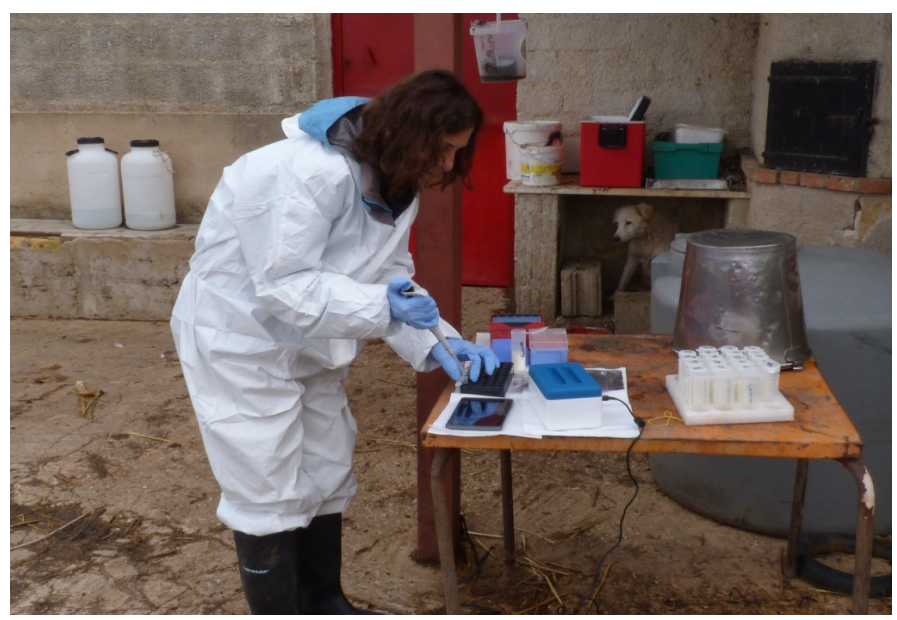

Figure 1. LAMP method in operation on farm laboratory diagnostics for sensitivity but much better in speed and handling. It also proved to be more robust to the inhibitory effect of milk components that affect amplification of the PCR.

The system showed a high specificity identifying only the M.agalactiae DNA and not that from the other animal mycoplasmas tested or controls just containing milk.

\section{Discussion}

These preliminary results show that the ICGene ${ }^{ø}$ system can be a practical and effective diagnostic tool for the diagnosis in the field of M.agalactiae pathogen. It can easily be adapted for the detection of other animal and human pathogens particularly for mycoplasma diseases like contagious bovine and caprine pleuropneumonia which occur in areas of the world where diagnostic laboratories are many kilometers from diagnostic laboratories. The innovative test is a significant improvement on traditional laboratory tests in terms of speed and robustness. Work is now continuing to take this test into the field and preliminary results look promising.

\section{References}

1. OIE (2012) Contagious agalactia. In: Manual of diagnostic tests and vaccines for terrestrial animals Seventh Ed., World Association for Animal Health, Paris 987-994.

2. Buonavoglia D, Fasanella A, Greco G, Pratelli A (1999) A study of an experimenta infection of sheep with Mycoplasma agalactiae. New Microbiol 22: 27-30. [Crossref]

3. Loria GR (2008) Contagious agalactia. In: Mycoplasma diseases of ruminants Nicholas R, Ayling RD and McAuliffe L. (Eds) CABI, UK 98-113.

4. Nicholas RAJ, Baker S (1998) Recovery of Mycoplasma from animals. In: Miles RJ and Nicholas RAJ (Editors) Mycoplasma protocols, Methods in molecular biology, vol. 104, Humana Press, Totowa, New Jersey, USA 37-43.

5. Tola S, Angioi A, Rocchigiani AM, Idini G, Manunta D, et al. (1997) Detection of Mycoplasma agalactiae in sheep milk samples by polymerase chain reaction.Vet Microbiol 54: 17-22. [Crossref]

6. McAuliffe L, Ellis RJ, Ayling RD, Nicholas RA (2003) Differentiation of Mycoplasma species by $16 \mathrm{~S}$ ribosomal DNA PCR and denaturing gradient gel electrophoresis fingerprinting. J Clin Microbiol 41: 4844-4847. [Crossref]

7. Postgate JR (1969) Viable count and viability. Methods in Microbiology 1: 611-628.

Copyright: (C2018 Loria GR. This is an open-access article distributed under the terms of the Creative Commons Attribution License, which permits unrestricted use, distribution, and reproduction in any medium, provided the original author and source are credited. 